\title{
is Research Square \\ CCL2 is associated with metastasis and poor prognosis of bladder cancer
}

wei gao

Shanghai Jiao Tong University

qiwang zhang

chizhou renmin hospital

lianhua zhang

Shanghai Jiao Tong University

qiang liu

Shanghai Jiao Tong University

xuehui duan

Shanghai Jiao Tong University

juanjie bo

Shanghai Jiao Tong University

guoliang yang ( $\nabla$ ygl0511@126.com)

Shanghai Jiao Tong University

Research article

Keywords: Bladder cancer, CCL2, Prognostic biomarker, Overall survival

Posted Date: December 11th, 2019

DOI: https://doi.org/10.21203/rs.2.18542/v1

License: (c) (i) This work is licensed under a Creative Commons Attribution 4.0 International License.

Read Full License 


\section{Abstract}

Background: Chemokine (C-C motif) ligand 2 (CCL2) is an important immune factor, which may be important in cancer progression by promoting proliferation, invasion metastasis and the tumor microenvironment. Recent researches have demonstrated that overexpression of CCL2 is associated with unfavorable prognosis in various cancer types. In this study, we aim to determine the prognostic value of CCL2 expression in patients with bladder cancer (BC).

Methods: We retrospectively enrolled 154 patients with bladder cancer at Renji Hospital, Shanghai Jiaotong University between 2005 and 2007. CCL2 expression was assessed by immunohistochemical staining and its association with clinicopathologic features and prognosis were evaluated. Kaplan-Meier method was applied to compare survival curves. Cox regression models were fitted to analyze the effect of prognostic factors on Overall survival (OS).

Results: CCL2 protein had high expression in 73 of 154 cases of BC (47\%). CCL2 overexpression was significantly associated with tumor grade $(P<0.001)$, stage $(P=0.005)$, and lymph node metastasis $(P=0.025)$. The Kaplan-Meier survival analysis demonstrated that CCL2 expression was significantly associated with DSS and OS (both $P<0.001$ ). Multivariate analysis further demonstrated that CCL2 was an independent prognostic factor for patients with BC.

Conclusion: CCL2 might be a new molecular marker to predict the prognosis of patients with BC. The novel risk classification based on combining CCL2 and TNM is more reliable than using either alone.

\section{Background}

Bladder cancer is the second most common genitourinary malignancy after prostate cancer in the USA. In the year 2016, the number of estimated new cases is 76,960 and number of estimated deaths from bladder cancer is 16,390[1]. At initial diagnosis, 75\% present with non-muscle-invasive bladder cancer (NMIBC) and can be managed with transurethral resection (TUR) and intravesical therapy. The remaining $25 \%$ present with muscle-invasive bladder cancer (MIBC) and the standard treatment is radical cystectomy $(\mathrm{RC})$. The main problems of NMIBC are recurrence and progression, while MIBC is frequently associated with metastatic disease and is the major cause of mortality. Despite improvement in surgical techniques, 5-year disease-free survival (DFS) and cancer-specific survival (CSS) after RC remains between 50 and $70 \%[2-4]$. Conventional clinicopathological parameters, such as tumor-node-metastasis (TNM), stage and grade of the tumor, as prognostic tools for patient counseling and treatment decisions. While these parameters have provided useful estimates of survival outcome, the heterogeneity of tumor biology leads to large variation in outcomes within each stage and grade. The integration of genetic, epigenetic, or protein biomarkers with these conventional prognostic models could provide more individualized risk stratification based on molecular characteristics of the tumor.

Chemokines are a superfamily of small, secreted molecules, which exert their activity by binding to Gprotein-coupled receptors. Chemokine (C-Cmotif) ligand 2 (CCL2), also referred to as monocyte 
chemoattractant protein-1 (MCP1), preferentially binds to the C-C chemokine receptor type 2 (CCR2), which is expressed in various tissues including thymus, lung, liver, kidney, pancreas and ovary[5]. CCL2 has been shown to play a critical role tumor cell generation and angiogenesis, and increase the chance of metastasis[6-9]. CCL2 is overexpressed in a variety of malignancies and is associated with adverse prognosis in breast, colorectal, prostate, cervix, thyroid cancer and renal cell carcinoma patients because of metastatic progression [10-15]. Previous studies showed that CCL2 were associated with TNM stage and grade in urine of bladder cancer[16], high levels of CCL2 expressed in bladder cancer mediates tumor invasion and is involved with advanced tumorigenesis[17]. In the present study, we aim to assess the expression of CCL2 and determine their prognostic value in BC patients.

\section{Methods}

\section{Patients}

For immunohistochemical assay, a total of 154 paraffin-embedded samples of transitional cell BC tissue were collected from our hospital between January 2005 and December 2007. The criteria for study enrollment were histopathological diagnosis of transitional cell carcinoma of the bladder, newly diagnosed and untreated, no history of other tumor, and the potential to follow up. We excluded carcinoma in situ from our study. For the use of these clinical materials for research purposes, prior patient's consent and approval from the Ethics Committee of our hospital. Clinical information about the samples is described in detail in Table 1. The patients included 115 males and 39 females from 35 to 80 years (mean age, 64.1 years). Tumor stage and grade were as follows: stage Ta-T1 $(n=94), T 2-T 4(n=$ $60)$, low grade $(n=55)$, high grade $(n=99)$. All patients underwent transurethral resection of the bladder tumor (TURBT), 60 patients underwent radical cystectomy. For the radical cystectomy patients, our surgeons performed a standard pelvic lymph node dissection (PLND) in $80 \%$ of patients, extended PLND in $13 \%$ of patients, and for various reasons $7 \%$ had limited. The reasons for limited PNLD during RC was that patients with older age and higher comorbidities were less likely to have a standard PLND. 18 patients had pathologic lymph node metastasis. Of the 154 patients examined, 41 patients died. The median follow-up time for overall survival (OS) was 61.5 months for patients at the time of analysis, and ranged from 7 to 118 months. Pathological staging and grading of each tumor were determined according to the 2009 tumor, node, metastasis staging system and the International Society of Urological Pathology 1998/World Health Organization 2004 classification, respectively. 
Table 1

Correlation between CCL2 expression and clinicopathologic features of the patients with $\mathrm{BC}$

\begin{tabular}{|c|c|c|c|c|}
\hline \multirow[t]{2}{*}{ Parameter } & \multirow[t]{2}{*}{ Case } & \multicolumn{2}{|c|}{ Expression of CCL2 } & \multirow[t]{2}{*}{$P$ value } \\
\hline & & LoI & & \\
\hline Gender & & & & 0.581 \\
\hline Female & 39 & 22 & 17 & \\
\hline Male & 115 & 59 & 56 & \\
\hline Age(years) & & & & 0.142 \\
\hline$\leq 65$ & 60 & 36 & 24 & \\
\hline$>65$ & 94 & 45 & 49 & \\
\hline Tumor size(cm) & & & & 0.616 \\
\hline$\leq 3$ & 96 & 52 & 44 & \\
\hline$>3$ & 58 & 29 & 29 & \\
\hline Tumor number & & & & 0.704 \\
\hline Unifocal & 84 & 43 & 41 & \\
\hline Multifocal & 70 & 38 & 32 & \\
\hline Grade & & & & $<0.001$ \\
\hline low & 55 & 40 & 15 & \\
\hline high & 99 & 41 & 58 & \\
\hline T stage & & & & 0.005 \\
\hline Ta- T1 & 94 & 58 & 36 & \\
\hline T2-T4 & 60 & 23 & 37 & \\
\hline Nodal status & & & & 0.025 \\
\hline pNO & 136 & 76 & 60 & \\
\hline pN1-N2 & 18 & 5 & 13 & \\
\hline
\end{tabular}

\section{Immunohistochemistry}


Formalin-fixed and paraffin embedded tissue sections $(5 \mathrm{~mm})$ were deparaffinized and rehydrated. Endogenous peroxidase activity was blocked with $0.3 \%$ hydrogen peroxide for $10 \mathrm{~min}$. Slides were incubated overnight at 48C in a humidified chamber with antibody against human CCL2 (ab9669, Abcam) at the dilution of 1:100. Biotinylated anti-rabbit link was used as secondary antibody (30 min). Slides were then incubated with a streptavidin-horse-radish peroxidase complex. Diaminobenzidine (DAB) was used as chromogen and the sections were counterstained with hematoxylin.

\section{Evaluation of immunohistochemistry}

In human BC tissue, CCL2 staining was detected in both tumor cells and stromal cells. The numbers of cells stained for CCL2 were counted and samples were categorized as "not stained (0-25\%)", "slightly stained (25-50\%)", "partially stained (50-75\%)", and "diffusely stained (75-100\%)" by one pathologist without prior knowledge of clinical features. In this study, "partially stained" and "diffusely stained" were defined as positive, and "not stained" and "slightly stained" were defined as negative. This cut point was set according to the previous publication[18].

\section{Statistical Analysis}

The significance of the relationships between CCL2 expression and clinicopathological parameters was evaluated using chi-squared tests. OS curves were calculated using the Kaplan-Meier method and compared by log-rank test. The significance of various variables for OS was analyzed by the Cox proportional hazards model in the multivariate analysis. SPSS 15.0 software (SPSS, Inc., Chicago, IL) was used for statistical analysis. A value of $<0.05$ was considered statistically significant.

\section{Results}

\section{Expression of CCL2 and its association with clinicopathological characteristics}

To determine whether the expression of CCL2 is associated with the development and progression of BC, we analyzed the expression of CCL2 by immunohistochemistry staining in 154 patients. CCL2 positive staining were mainly located in the cytoplasm of the tumor cells (Fig. 1). 47\% (73/154) of the tumor tissues were scored as high CCL2 expression. Clinicopathologic features for the patients in this research are listed in Table 1. High levels of CCL2 protein expression were significantly correlated with tumor grade and stage $(P<0.001$ and $P=0.005$, respectively). High CCL2 expression also correlated with lymph node metastasis $(P=0.025)$ However, CCL2 protein expression was not associated with other clinicopathological features such as age, sex, tumor size, and tumor number.

\section{High expression of CCL2 is associated with poor prognosis}


Using Kaplan-Meier analysis method, we observed that the expression of CCL2 in BC was significantly correlated with DSS and OS $(P<0.001$, Table 2$)$. The log-rank test further demonstrated that the survival time was significantly different between groups with high and low expression of CCL2 protein, indicating that high level of CCL2 was tightly correlated with a shorter survival (Fig. 2). On multivariate analysis, high CCL2 expression is an independent prognostic factor in both for DSS and OS (HR $=5.423 \mathrm{P}=0.001$ and $\mathrm{HR}=2.589 \mathrm{P}=0.008$ ). After backward selection, stage, and CCL2 expression remained as independent prognostic factors for DSS, but age, stage, CCL2 expression and lymph node metastasis as independent prognostic factors for OS (Table 3). 
Table 2

Univariate survival analysis of disease-specific survival and overall survival in 154 patients with $\mathrm{BC}$

\begin{tabular}{|c|c|c|c|c|c|}
\hline \multirow[t]{2}{*}{ Variable } & \multirow[t]{2}{*}{ case } & \multicolumn{2}{|c|}{ Disease-specific survival } & \multicolumn{2}{|c|}{ Overall survival } \\
\hline & & Mean $\pm S E$ & $P$ value & Mean \pm SE & $P$ value \\
\hline Gender & & & 0.969 & & 0.892 \\
\hline Female & 39 & $99 \pm 6$ & & $92 \pm 6$ & \\
\hline Male & 115 & $100 \pm 3$ & & $95 \pm 4$ & \\
\hline Age(yeas) & & & 0.074 & & 0.014 \\
\hline$\leq 65$ & 60 & $106 \pm 4$ & & $104 \pm 4$ & \\
\hline$>65$ & 94 & $92 \pm 4$ & & $85 \pm 4$ & \\
\hline Tumor number & & & 0.033 & & 0.119 \\
\hline Unifocal & 84 & $105 \pm 3$ & & $99 \pm 4$ & \\
\hline Mutlifocal & 70 & $91 \pm 5$ & & $87 \pm 5$ & \\
\hline Tumor size & & & 0.471 & & 0.524 \\
\hline$\leq 3$ & 96 & $101 \pm 4$ & & $96 \pm 4$ & \\
\hline$>3$ & 58 & $95 \pm 5$ & & $89 \pm 5$ & \\
\hline Grade & & & $<0.001$ & & $<0.001$ \\
\hline Low & 55 & $115 \pm 2$ & & $113 \pm 2$ & \\
\hline High & 99 & $89 \pm 4$ & & $79 \pm 5$ & \\
\hline T stage & & & $<0.001$ & & $<0.001$ \\
\hline Ta-T1 & 94 & $115 \pm 2$ & & $108 \pm 3$ & \\
\hline T2-T4 & 60 & $75 \pm 6$ & & $70 \pm 6$ & \\
\hline CCL2 expression & & & $<0.001$ & & $<0.001$ \\
\hline Low & 81 & $113 \pm 2$ & & $106 \pm 3$ & \\
\hline High & 73 & $83 \pm 5$ & & $78 \pm 5$ & \\
\hline Nodal status & & & $<0.001$ & & $<0.001$ \\
\hline pNO & 136 & $105 \pm 3$ & & $99 \pm 3$ & \\
\hline pN1-N2 & 18 & $41 \pm 5$ & & $39 \pm 6$ & \\
\hline
\end{tabular}


Table 3

Multivariate cox model analysis of disease-specific survival and overall survival

\begin{tabular}{|lllll|}
\hline Variable & Disease-specific survival & Overall survival & \\
\cline { 2 - 4 } & Hazard ratio (95\% Cl) & P value & Hazard ratio (95\% Cl) & P value \\
\hline Gender & & 0.363 & & 0.233 \\
\hline Female vs $>$ Male & $0.669(0.282-1.590)$ & & $0.643(0.311-1.328)$ & \\
\hline Age (years) & & 0.194 & & 0.048 \\
\hline$\leq 65$ vs $>65$ & $1.766(0.749-4.165)$ & & $2.144(1.007-4.438)$ & \\
\hline Tumor number & & 0.099 & & 0.451 \\
\hline unifocal vs mutlifocal & $1.946(0.883-4.289)$ & & $1.294(0.662-2.529)$ & \\
\hline Tumor size & & 0.433 & & 0.422 \\
\hline$\leq 3$ cm vs $>3$ cm & $0.728(0.329-1.610)$ & & $0.758(0.386-1.490)$ & \\
\hline Grade & & 0.991 & & 0.086 \\
\hline Low vs High & $0.988(0.136-7.199)$ & & $2.950(0.857-10.152)$ & \\
\hline T stage & & 0.002 & & 0.009 \\
\hline Ta-T1 vs T2-T4 & $10.703(2.462-47.221)$ & & $3.193(1.342-7.598)$ & \\
\hline CCL2 expression & & 0.001 & & 0.008 \\
\hline Low vs High & $5.423(1.992-14.765)$ & & $2.589(1.277-5.252)$ & \\
\hline Nodal status & & 0.143 & & \\
\hline pN0 vs pN1-N2 & $1.836(0.814-4.139)$ & & $2.563(1.173-5.600)$ & \\
\hline
\end{tabular}

\section{Discussion}

To our knowledge, this study is the first to report an association between high CCL2 expression and a greater risk of death and metastasis in bladder cancer. In this study, we first showed that CCL2 expression was correlated with tumor stage, worse tumor grade, metastasis and poor prognosis in BC patients.

Chemokines and their receptors mediate acute inflammation and were initially described in the context of their chemoattractant function for leukocytes; chemokines, induced at sites of inflammation, provide directional cues during migration of leukocytes to damaged or infected tissues[19]. However, elevated expression of chemokines, leading to alterations in chemokine-receptor signaling can contribute to chronic inflammation and malignancy[20,21]. Cancer cells and host stromal cells in the tumor microenvironment including endothelial cells, fibroblasts, mesenchymal stem cells and infiltrating 
leukocytes produce a wide range of chemokines that exert numerous biological functions during tumor progression and metastasis [22, 23]. It is reported that an elevated expression of CCL2 and CCR2 is observed in a variety of malignancies and is associated with adverse prognosis in patients with breast, nasopharyngeal, colorectal, prostate and pancreatic cancer [24-27]. Blocking CCL2/CCR2 signaling pathway may serve as a novel strategy to help patients with certain kinds of cancers[28].

In our study, we demonstrated the elevated CCL2 levels significantly correlated with lymph node metastasis. We found that CCL2 expression was inversely correlated with OS. The patients with higher expression of CCL2 had a shorter survival. Gastric cancer patients with high CCL2 expression also had a lower overall survival rate, elevated serum and intratumoral CCL2 levels significantly correlated with lymph node metastasis [29,30], suggesting CCL2 to be a prognostic marker for gastric cancer[31]. Similarly, in patients with primary and metastatic colorectal cancer, CCL2 levels were elevated in serum, and increased with progressive Dukes' stages[25] and neoplastic progression [32]. CCL2 was further implicated as a prognostic marker and an independent predictor of liver metastasis in colorectal cancer patients[33].

Previous studies have demonstrated that inflammatory environment, which primarily consists of inflammatory cells and inflammatory cytokines, is involved in cancer progression and metastasis[34-36]. Among the inflammatory cells, macrophages are especially abundant and could be observed at the entire period of tumor progression[37]. Blocking CCL2-CCR2 signaling inhibits macrophage infiltration, delays breast cancer metastasis and prolongs the survival of tumor-bearing mice[9]. Apart from recruiting and educating TAMs, CCL2/CCR2 has been reported to be responsible for the accumulation of myeloidderived suppressor cells (MDSCs) in tumor sites [32,38]. Another study suggested that CCL2/CCR2 axis could promote cancer metastasis by up-regulation of MMP2/9 through ERK1/2 signaling pathway [24]. However, these studies were performed in other cancer models, the mechanism that CCL2 contributes to the unfavorable outcomes of $\mathrm{BC}$ patients remains to be fully understood.

There are several limitations of this study that warrant further discussion. First, because all of the patients enrolled in our research are from Asia, the result of this study needs to be validated in other populations and larger cohorts. Second, the predictive value of CCL2 expression is simply verified in BC owing to limited cases with lymph node metastasis. Third, this is a retrospective analysis. Further assessment in more lymph node tissues need to be performed in the future.

\section{Conclusion}

We have identified the increased expression of CCL2 in BC as an independent unfavorable prognostic factor, which could be integrated with pathologic $T$ stage, grade and the lymph node of the tumor to generate a nomogram to give a better risk stratification for patients with different prognosis. However, functional studies are needed to elucidate the biological mechanisms behind this association.

\section{Abbreviations}


CCL2

Chemokine (C-C motif) ligand 2

$\mathrm{BC}$

bladder cancer

OS

Overall survival

NMIBC

non-muscle-invasive bladder cancer

TUR

transurethral resection

MIBC

muscle-invasive bladder cancer

$\mathrm{RC}$

radical cystectomy

DFS

disease-free survival

CSS

cancer-specific survival

MDSCs

myeloid-derived suppressor cells

\section{Declarations}

\section{Ethics approval and consent to participate}

This study was conducted with the approval of the medical ethics committee of Renji Hospital Shanghai Jiao Tong University School of Medicine. Each patient signed a written informed consent form before entry into this study.

\section{Consent for publication}

Not applicable.

\section{Competing interests}

The authors declare that they have no competing interests.

\section{Acknowledgements}

Not applicable.

\section{Funding}


This study was supported by grants from Shanghai Health Bureau (No.20164Y0124), National Natural Science Foundation of China (81902561), Incubating Program for clinical Research and Innovation of Renji Hospital Shanghai Jiao Tong University School of Medicine (PYZY16-008, PYXJS16-015).

\section{References}

[1] Siegel RL, Miller KD, Jemal A. Cancer statistics, 2016. CA: a cancer journal for clinicians. 2016;66:7-30.

[2] Ghoneim MA, Abdel-Latif M, el-Mekresh M, Abol-Enein H, Mosbah A, Ashamallah A, et al. Radical cystectomy for carcinoma of the bladder: 2,720 consecutive cases 5 years later. The Journal of urology. 2008;180:121-7.

[3] Shariat SF, Karakiewicz PI, Palapattu GS, Lotan Y, Rogers CG, Amiel GE, et al. Outcomes of radical cystectomy for transitional cell carcinoma of the bladder: a contemporary series from the Bladder Cancer Research Consortium. The Journal of urology. 2006;176:2414-22; discussion 22.

[4] Stein JP, Lieskovsky G, Cote R, Groshen S, Feng AC, Boyd S, et al. Radical cystectomy in the treatment of invasive bladder cancer: long-term results in 1,054 patients. Journal of clinical oncology : official journal of the American Society of Clinical Oncology. 2001;19:666-75.

[5] Lim SY, Yuzhalin AE, Gordon-Weeks AN, Muschel RJ. Targeting the CCL2-CCR2 signaling axis in cancer metastasis. Oncotarget. 2016;7:28697-710.

[6] Lin ZY, Chuang YH, Chuang WL. Cancer-associated fibroblasts up-regulate CCL2, CCL26, IL6 and LOXL2 genes related to promotion of cancer progression in hepatocellular carcinoma cells. Biomedicine \& pharmacotherapy $=$ Biomedecine $\&$ pharmacotherapie. 2012;66:525-9.

[7] Fang WB, Jokar I, Zou A, Lambert D, Dendukuri P, Cheng N. CCL2/CCR2 chemokine signaling coordinates survival and motility of breast cancer cells through Smad3 protein- and p42/44 mitogenactivated protein kinase (MAPK)-dependent mechanisms. The Journal of biological chemistry. 2012;287:36593-608.

[8] Kudo-Saito C, Shirako H, Ohike M, Tsukamoto N, Kawakami Y. CCL2 is critical for immunosuppression to promote cancer metastasis. Clinical \& experimental metastasis. 2013;30:393-405.

[9] Qian BZ, Li J, Zhang H, Kitamura T, Zhang J, Campion LR, et al. CCL2 recruits inflammatory monocytes to facilitate breast-tumour metastasis. Nature. 2011;475:222-5.

[10] Soria G, Ofri-Shahak M, Haas I, Yaal-Hahoshen N, Leider-Trejo L, Leibovich-Rivkin T, et al. Inflammatory mediators in breast cancer: coordinated expression of TNFalpha \& IL-1 beta with CCL2 \& CCL5 and effects on epithelial-to-mesenchymal transition. BMC cancer. 2011;11:130.

[11] Yoshidome H, Kohno H, Shida T, Kimura F, Shimizu H, Ohtsuka M, et al. Significance of monocyte chemoattractant protein-1 in angiogenesis and survival in colorectal liver metastases. International 
journal of oncology. 2009;34:923-30.

[12] Zhang J, Patel L, Pienta KJ. CC chemokine ligand 2 (CCL2) promotes prostate cancer tumorigenesis and metastasis. Cytokine \& growth factor reviews. 2010;21:41-8.

[13] Zijlmans HJ, Fleuren GJ, Baelde HJ, Eilers PH, Kenter GG, Gorter A. The absence of CCL2 expression in cervical carcinoma is associated with increased survival and loss of heterozygosity at 17q11.2. The Journal of pathology. 2006;208:507-17.

[14] Tanaka K, Kurebayashi J, Sohda M, Nomura T, Prabhakar U, Yan L, et al. The expression of monocyte chemotactic protein-1 in papillary thyroid carcinoma is correlated with lymph node metastasis and tumor recurrence. Thyroid : official journal of the American Thyroid Association. 2009;19:21-5.

[15] Yang Y, Zhai C, Chang Y, Zhou L, Shi T, Tan C, et al. High expression of chemokine CCL2 is associated with recurrence after surgery in clear-cell renal cell carcinoma. Urologic oncology. 2016;34:238 e19-26.

[16] Amann B, Perabo FG, Wirger A, Hugenschmidt H, Schultze-Seemann W. Urinary levels of monocyte chemo-attractant protein-1 correlate with tumour stage and grade in patients with bladder cancer. British journal of urology. 1998;82:118-21.

[17] Chiu HY, Sun KH, Chen SY, Wang HH, Lee MY, Tsou YC, et al. Autocrine CCL2 promotes cell migration and invasion via PKC activation and tyrosine phosphorylation of paxillin in bladder cancer cells. Cytokine. 2012;59:423-32.

[18] Arakaki R, Yamasaki T, Kanno T, Shibasaki N, Sakamoto H, Utsunomiya N, et al. CCL2 as a potential therapeutic target for clear cell renal cell carcinoma. Cancer medicine. 2016;5:2920-33.

[19] Zlotnik A, Yoshie O, Nomiyama H. The chemokine and chemokine receptor superfamilies and their molecular evolution. Genome biology. 2006;7:243.

[20] Zlotnik A. Chemokines and cancer. International journal of cancer. 2006;119:2026-9.

[21] Balkwill F. Cancer and the chemokine network. Nature reviews Cancer. 2004;4:540-50.

[22] Balkwill FR. The chemokine system and cancer. The Journal of pathology. 2012;226:148-57.

[23] Lazennec G, Richmond A. Chemokines and chemokine receptors: new insights into cancer-related inflammation. Trends in molecular medicine. 2010;16:133-44.

[24] Yang J, Lv X, Chen J, Xie C, Xia W, Jiang C, et al. CCL2-CCR2 axis promotes metastasis of nasopharyngeal carcinoma by activating ERK1/2-MMP2/9 pathway. Oncotarget. 2016;7:15632-47.

[25] Zhao L, Lim SY, Gordon-Weeks AN, Tapmeier TT, Im JH, Cao Y, et al. Recruitment of a myeloid cell subset (CD11b/Gr1 mid) via CCL2/CCR2 promotes the development of colorectal cancer liver metastasis. Hepatology. 2013;57:829-39. 
[26] Lu Y, Chen Q, Corey E, Xie W, Fan J, Mizokami A, et al. Activation of MCP-1/CCR2 axis promotes prostate cancer growth in bone. Clinical \& experimental metastasis. 2009;26:161-9.

[27] Monti P, Leone BE, Marchesi F, Balzano G, Zerbi A, Scaltrini F, et al. The CC chemokine MCP-1/CCL2 in pancreatic cancer progression: regulation of expression and potential mechanisms of antimalignant activity. Cancer research. 2003;63:7451-61.

[28] Huang B, Lei Z, Zhao J, Gong W, Liu J, Chen Z, et al. CCL2/CCR2 pathway mediates recruitment of myeloid suppressor cells to cancers. Cancer letters. 2007;252:86-92.

[29] Tonouchi H, Miki C, Tanaka K, Kusunoki M. Profile of monocyte chemoattractant protein-1 circulating levels in gastric cancer patients. Scandinavian journal of gastroenterology. 2002;37:830-3.

[30] Futagami S, Tatsuguchi A, Hiratsuka T, Shindo T, Horie A, Hamamoto T, et al. Monocyte chemoattractant protein 1 and CD40 ligation have a synergistic effect on vascular endothelial growth factor production through cyclooxygenase 2 upregulation in gastric cancer. Journal of gastroenterology. 2008;43:216-24.

[31] Tao LL, Shi SJ, Chen LB, Huang GC. Expression of monocyte chemotactic protein-1/CCL2 in gastric cancer and its relationship with tumor hypoxia. World journal of gastroenterology. 2014;20:4421-7.

[32] Chun E, Lavoie S, Michaud M, Gallini CA, Kim J, Soucy G, et al. CCL2 Promotes Colorectal Carcinogenesis by Enhancing Polymorphonuclear Myeloid-Derived Suppressor Cell Population and Function. Cell reports. 2015;12:244-57.

[33] Hu H, Sun L, Guo C, Liu Q, Zhou Z, Peng L, et al. Tumor cell-microenvironment interaction models coupled with clinical validation reveal CCL2 and SNCG as two predictors of colorectal cancer hepatic metastasis. Clinical cancer research : an official journal of the American Association for Cancer Research. 2009;15:5485-93.

[34] Coussens LM, Werb Z. Inflammation and cancer. Nature. 2002;420:860-7.

[35] Grivennikov SI, Greten FR, Karin M. Immunity, inflammation, and cancer. Cell. 2010;140:883-99.

[36] Kim S, Takahashi H, Lin WW, Descargues P, Grivennikov S, Kim Y, et al. Carcinoma-produced factors activate myeloid cells through TLR2 to stimulate metastasis. Nature. 2009;457:102-6.

[37] Noy R, Pollard JW. Tumor-associated macrophages: from mechanisms to therapy. Immunity. 2014;41:49-61.

[38] Chang AL, Miska J, Wainwright DA, Dey M, Rivetta CV, Yu D, et al. CCL2 Produced by the Glioma Microenvironment Is Essential for the Recruitment of Regulatory T Cells and Myeloid-Derived Suppressor Cells. Cancer research. 2016;76:5671-82. 